\title{
Nematic order of model goethite nanorods in a magnetic field
}

\author{
H. H. Wensink and G. J. Vroege* \\ Van 't Hoff Laboratory for Physical and Colloid Chemistry, Debye Institute, Utrecht University, Padualaan 8, 3584 CH Utrecht, \\ The Netherlands
}

(Received 17 January 2005; revised manuscript received 4 March 2005; published 21 September 2005)

\begin{abstract}
We explore the nematic order of model goethite nanorods in an external magnetic field within OnsagerParsons density functional theory. The goethite rods are represented by monodisperse, charged spherocylinders with a permanent magnetic moment along the rod main axis, forcing the particles to align parallel to the magnetic field at low field strength. The intrinsic diamagnetic susceptibility anisometry of the rods is negative, which leads to a preferred perpendicular orientation at higher field strength. It is shown that these competing effects may give rise to intricate phase behavior, including a pronounced stability of biaxial nematic order and the presence of reentrant phase transitions and demixing phenomena.
\end{abstract}

DOI: 10.1103/PhysRevE.72.031708

PACS number(s): 61.30.Gd, 64.70.Md, 82.70.Dd

\section{INTRODUCTION}

The effect of external fields on the phase behavior of anisometric colloids (rods and plates) has received considerable attention over the last years, in particular in the domain of density functional theory [1]. The presence of an interface or wall breaks the translational and orientational symmetry and leads to local structures that are drastically different from those found in the bulk [2-5]. Macroscopically different behavior may be brought about for instance by a gravitational field $[6,7]$. If the buoyant mass of the colloids is sufficiently high, inhomogeneous density profiles are built up along the vertical dimension of the system. In some cases, this may lead to the formation of multiple phase equilibria not encountered at zero field [8-10].

In addition, much effort has been put into investigating the behavior of anisometric colloids in an applied electric or magnetic field. Owing to the fact that the electric polarizability (or magnetic susceptibility) is different along the short and long axes of the particle, electric or magnetic dipole moments are induced which give rise to an additional energetic contribution to the free energy. The resulting competition between minimal self-energy and maximal configurational entropy of the rods drastically changes the orientational structure of the system and leads to qualitatively different phase behavior. An important difference with the previously mentioned class of fields is that an external electric or magnetic field is only coupled with the orientational degrees of freedom of the rods or plates and, therefore, does not directly lead to spatial inhomogeneities.

A systematic attempt to incorporate the effect of these directional external fields into the classic Onsager theory [11] for lyotropic anisometric particles has been reported by Khokhlov and Semenov [12]. Within a simplified variational approach, general phase behavior scenarios were presented for both hard and semiflexible rod systems subjected to various types of directional fields. Later on, similar studies have

\footnotetext{
*Corresponding author.
}

Electronic address: g.j.vroege@chem.uu.nl been carried out within a numerical treatment of the Onsager theory [13,14] and Lee-Parsons [15] and modified weighteddensity approximation (MWDA) -type [16] density functional approaches. In the latter case, various (spatially) inhomogeneous liquid crystal states which may occur at high packing fractions, e.g., smectic and (plastic) solids, were also considered.

Although the experimental study of electromagnetic field effects on colloidal suspensions has been pioneered a long time ago (see Ref. [17] and references therein), the topic has been the subject of renewed interest because of recent experiments on colloidal goethite $(\alpha-\mathrm{FeOOH})$ suspensions $[17,18]$. These systems consist of charged, bar-shaped nanorods with peculiar magnetic properties. These particles not only possess a permanent magnetic moment directed along their longitudinal axis, originating from uncompensated surface spins within the antiferromagnetic crystal lattice, but also an enhanced magnetic susceptibility along their short axes. This means that additional magnetic moments are induced perpendicular to the main axis upon applying an external field. These unique properties become manifest in particular in concentrated, nematic suspensions subjected to magnetic fields below $1 \mathrm{~T}$. At low field strengths, the induced moments are weak and the permanent ones give rise to enhanced nematic alignment along the field direction. However, at high field strengths, the induced moments are dominant and cause the rods to orient with their longitudinal axes perpendicular to the field. The associated reorientation of the nematic director can be clearly observed from x-ray scattering measurements [19].

Although the realignment phenomenon can be understood directly from the counteracting effects of the permanent and induced magnetic dipoles, very little is known about the overall phase diagram of goethite systems as a function of applied field strength. In Ref. [18], a first attempt has been made toward a global understanding of the phase behavior, but the analysis there was restricted to ideal systems, described by a simple Boltzmann distribution for the rod orientations, and weakly correlated systems described by an expansion of the Onsager free energy up to first order in the degree of nematic order. Both approaches may be used for 
very dilute isotropic suspensions where particle interactions are not too important, but lack predictive power for dense systems where interactions lead to strong deviations from the ideal Boltzmann orientation distribution associated with the magnetic energy.

In this paper, we give a full numerical analysis of the phase behavior of model goethite suspensions, starting from Onsager-Parsons density functional theory. The numerical approach not only allows us to explore the full nematic density range, it also provides a reliable way to probe subtle changes in the nematic orientation symmetry as a function of the applied field strength, in particular transitions between uniaxial and biaxial nematic states. We stress that the model goethite system we consider in this paper is a strongly simplified one. The particles are considered as monodisperse, charged spherocylinders interacting via electrostatic repulsions. Weak attractive van der Waals forces are present in the experimental systems [20], but are difficult to incorporate theoretically and are not considered here. The interaction energy between the total dipole moments on the rods is estimated to be of the order $10^{-5} k_{B} T$ [20] and, therefore, can be safely neglected. Moreover, the particles' considerable size polydispersity (along all three particle axes) not only leads to a wide variety of particle shapes, but also a strong concomitant spread in the magnetic and electrostatic properties (e.g., surface charge). Therefore, all quantities presented here pertaining to the electrostatic and magnetic properties should be considered as typical values rather than quantitative averages.

This paper is constructed as follows. In Sec. II, the Onsager-Parsons approach will be presented and adapted for charged particles in the presence of a directional field. In Sec. III, we quantify the average magnetic and electrostatic properties of the goethite rods. Depending on the relative contribution of the (average) permanent and induced dipole moments, several phase diagram scenarios for goethite were constructed. They will be discussed in Sec. V. Finally, in Sec. VI, we summarize our findings and provide a brief outlook for future work.

\section{ONSAGER-PARSONS THEORY}

The starting point of our analysis is the magnetic energy of a single goethite rod which consists of two parts: (i) a contribution for the remanent magnetic moment along the main rod axis, linear in the magnetic field strength $B$; and (ii) one representing the induced magnetization perpendicular to the main axis, which depends quadratically on $B$. Following Ref. [18], the total magnetic energy can be written as

$$
\beta U_{m}(\cos \theta)=-J B \mathcal{P}_{1}(\cos \theta)+K B^{2} \mathcal{P}_{2}(\cos \theta)
$$

in terms of the Legendre polynomials $\mathcal{P}_{n}$ with $\theta$ being the angle between the main rod axis and the direction of the magnetic field. The quantities $J$ and $K$, with dimensions given in $\mathrm{T}^{-1}$ and $\mathrm{T}^{-2}$ (Tesla), respectively, are related to the (average) remanent dipole moment $\mu_{r}$ and the diamagnetic susceptibility anisometry $\Delta \chi=\chi_{\|}-\chi_{\perp}<0$ of the nanorods via

$$
J=\beta \mu_{r}, \quad K=\beta|\Delta \chi| v_{0} / 3 \mu_{0}
$$

with $v_{0}$ the rod volume and $\mu_{0}$ the magnetic permeability in a vacuum. All quantities will be specified in Sec. III A.

As a first approximation, the bar-shaped goethite rods are modeled as (uniaxial) spherocylinders with equal length $L$ and diameter $D$, bearing a uniform electric surface charge. Following Onsager [11], the charged rods interact via an effective hard core repulsion, characterized by an effective diameter $D_{\text {eff }}>D$ which depends on the charge density on the particle and the ionic strength of the solvent. The OnsagerParsons free energy of the system in the presence of an external directional field can be cast into the following functional form $[11,21]$ :

$$
\frac{\beta F}{N} \sim \ln c+\sigma[f]+c g_{\mathrm{P}}(\phi)\{\rho[f]+h \eta[f]\}+\left\langle\beta U_{m}(\cos \theta)\right\rangle_{f},
$$

where the brackets denote an orientational average according to some singlet orientation distribution function (ODF) $f(\Omega)$ characterizing the average orientational configuration of the system in terms of the solid angle $\Omega$. Here, $c$ and $\phi$ denote the effective (dimensionless) number density $c$ $=(\pi / 4) N L^{2} D_{\text {eff }} / V$ and packing fraction $\phi=N v_{\text {eff }} / V$ with $v_{\text {eff }}=(\pi / 4) L D_{\text {eff }}^{2}+(\pi / 6) D_{\text {eff }}^{3}$ being the effective volume of the spherocylinder. The last term in Eq. (3) represents the external magnetic contribution [cf. Eq. (1)], whereas $\sigma$ and $\rho$ quantify the orientational and packing entropy, respectively, defined by the following angular averages:

$$
\begin{gathered}
\sigma[f]=\langle\ln 4 \pi f(\Omega)\rangle_{f}, \\
\rho[f]=\frac{4}{\pi}\left\{\left\langle\left\langle\sin \gamma\left(\Omega, \Omega^{\prime}\right)\right\rangle\right\rangle_{f}+\frac{\pi D_{\text {eff }}}{L}+\frac{2 \pi}{3}\left(\frac{D_{\text {eff }}}{L}\right)^{2}\right\},
\end{gathered}
$$

where $\gamma$ is the angle between two spherocylinders with orientations $\Omega$ and $\Omega^{\prime}$. The second term in $\rho[f]$ arises from end-cap contributions to the (electrostatic) repulsion between two short spherocylinders and is strictly speaking only valid in the isotropic state [22]. The $O\left((D / L)^{2}\right)$ contribution has been included to retain the excluded volume of a hard spherocylinder in the limit $D_{\text {eff }} \rightarrow D$. The contribution $\eta$ expresses the so-called "twisting effect" arising from the orientation-dependent nature of the electrostatic interaction $[11,23]$

$$
\begin{aligned}
\eta[f]= & \frac{4}{\pi}\left\langle\left\langle-\sin \gamma\left(\Omega, \Omega^{\prime}\right) \ln \left[\sin \gamma\left(\Omega, \Omega^{\prime}\right)\right]\right\rangle\right\rangle_{f} \\
& -(\ln 2-1 / 2) \rho[f] .
\end{aligned}
$$

The importance of this effect is quantified by a twisting parameter $h=\kappa^{-1} / D_{\text {eff }}$, defined as the ratio of the Debye screening length $\kappa^{-1}$ and the effective rod diameter [21]. All properties pertaining to the electrostatic interactions will be specified in Sec. III B for the case of goethite. The function $g_{P}(\phi)$ originates from the Lee-Parsons [24-26] rescaling of the original second virial theory. In its simplest form, $g_{P}(\phi)$ is proportional to the Carnahan-Starling excess free energy $\mathcal{F}_{C S}^{e x}$ (in units $k_{B} T$ per particle) for a hard sphere fluid [27] 


$$
g_{P}(\phi)=\frac{\mathcal{F}_{C S}^{e x}}{4 \phi}=\frac{1-(3 / 4) \phi}{(1-\phi)^{2}}
$$

in terms of the effective rod volume fraction $\phi$. As in Eq. (4), we implicitly assume here that a charged spherocylinder can be represented by a hard spherocylinder with an effective diameter $D_{\text {eff }}$.

The equilibrium ODF is determined by applying a formal minimization of the free energy. This yields the following self-consistency condition:

$$
\begin{aligned}
f(\Omega)= & Z \exp \left[-\frac{8}{\pi} \operatorname{cg}_{P}(\phi) \int \omega\left(\Omega, \Omega^{\prime}\right) f\left(\Omega^{\prime}\right) d \Omega^{\prime}\right] \\
& \times \exp \left[-\beta U_{\mathrm{m}}(\cos \theta)\right],
\end{aligned}
$$

where $Z$ is obtained from the normalization condition $\int f(\Omega) d \Omega=1 . \omega$ is the orientation-dependent part of the second virial coefficient for two charged spherocylinders at fixed solid angles $\Omega$ and $\Omega^{\prime}$ [21]

$$
\begin{aligned}
\omega\left(\Omega, \Omega^{\prime}\right)= & \sin \gamma\left(\Omega, \Omega^{\prime}\right) \times\left(1-h\left\{\ln \left[\sin \gamma\left(\Omega, \Omega^{\prime}\right)\right]\right.\right. \\
& +\ln 2-1 / 2\}) .
\end{aligned}
$$

The solid angle $\Omega$ is conveniently parametrized in terms of a polar angle $0 \leqslant \theta \leqslant \pi$ and an azimuthal one $0 \leqslant \varphi \leqslant 2 \pi$, so that $d \Omega=\sin \theta d \theta d \varphi$. Throughout the remainder of this text, $\theta$ always refers to the angle between the rod main axis and the direction of the magnetic field. The azimuthal angle $\varphi$, then describes the projection of the rod axis onto the plane perpendicular to the field. Equation (7) is solved iteratively according to a discretization scheme outlined in Ref. [28] using a grid of angles $\left\{\theta_{i}, \varphi_{j}\right\}_{i, j=1, N}$ of mesh size $N=30$ for the biaxial state and $\left\{\theta_{i}\right\}_{i=1, N}(N=100)$ for the uniaxial state. Refining these grid sizes did not lead to significant changes in the results.

Once the ODF has been obtained, the thermodynamics and phase behavior of the system can be inferred from the osmotic pressure and chemical potential. These are conveniently expressed in terms of the parameters $\sigma, \rho$, and $\eta$ [cf. Eqs. (4) and (5)] via

$$
\begin{aligned}
\beta \widetilde{\Pi}= & c+c^{2} \frac{\partial \phi g_{P}}{\partial \phi}\{\rho[f]+h \eta[f]\}, \\
\beta \widetilde{\mu}= & \ln c+\sigma[f]+2 c\left(g_{P}+\frac{\phi}{2} \frac{\partial g_{P}}{\partial \phi}\right)\{\rho[f] \\
& +h \eta[f]\}+\left\langle\beta U_{m}(\cos \theta)\right\rangle_{f},
\end{aligned}
$$

with $\beta \tilde{\Pi}$ and $\beta \tilde{\mu}$ denoting the dimensionless pressure and chemical potential, respectively. At phase coexistence, these quantities must be equal in each of the coexisting phases. Second-order phase transitions from, e.g., uniaxial to biaxial nematic symmetries can be localized by means of a firstorder bifurcation analysis, as discussed in detail in Ref. [29].

\section{Nematic order parameters}

To specify the orientational symmetry in the various nematic states, we calculate several nematic order parameters.
The degree of dipolar order due to the permanent dipoles at finite field strengths is given by the following average

$$
S_{1}=\left\langle\mathcal{P}_{1} \cos (\theta)\right\rangle_{f} .
$$

Note that for nondipolar rods $S_{1} \equiv 0$ and $f(\Omega)$ is invariant with respect to the inversion $\theta \rightarrow(\pi-\theta)$. In principle, the (quadrupolar) orientational order around the field direction could be expressed in terms of higher order (associated) Legendre polynomials depending on $\theta$ and $\varphi$. However, the average direction of the rods may be different from the field direction, in particular at higher field strengths where the rods tend to be perpendicular to the field, so that the nematic director is not always known a priori. Therefore, it is advantageous to analyze the orientational order from the symmetric, second-rank tensor $\mathbf{Q}$, defined as [30]

$$
\mathbf{Q}=\frac{3}{2}\langle\hat{\mathbf{u}} \otimes \hat{\mathbf{u}}\rangle_{f}-\frac{1}{2} \mathbf{I},
$$

where $\hat{\mathbf{u}}=\{\sin \theta \cos \varphi, \sin \theta \sin \varphi, \cos \theta\}$ is the orientation unit vector of the rod, $\otimes$ denotes the dyadic product, and $\mathbf{I}$ is the second-rank unit tensor. The nematic order parameter $S_{2}$ is defined as the largest eigenvalue of $\mathbf{Q}$ and the nematic director is the corresponding eigenvector. The sign of $S_{2}$ allows one to distinguish two types of nematic order. First, if $0<S_{2} \leqslant 1$, the particles are preferentially aligned along the principal director, corresponding to common polar (or prolate) nematic order. Alternatively, if $-1 / 2 \leqslant S_{2}<0$, the particles are mainly oriented in a plane perpendicular to the director, leading to planar (i.e., oblate or antinematic) order. Note that the latter type of nematic order only occurs in systems subjected to disorientational external fields and is not stable at zero-field conditions [12].

To discriminate between uniaxial and biaxial nematic states, we focus on the two smallest eigenvalues of $\mathbf{Q}$. If they are degenerate, all directions perpendicular to the director are equally probable and the system is of uniaxial symmetry. In the biaxial nematic state, the difference $\Delta$ between these eigenvalues provides a measure for the degree of preferred order along a secondary director perpendicular to the main one.

\section{INTRINSIC ROD PROPERTIES}

\section{A. Magnetic properties}

The electronic and magnetic properties of the goethite rods have been extensively discussed in Ref. [18]. Here, we shall only briefly recall some of the basic quantities we need as input for our calculations. First of all, the remanent magnetic dipole moment of the rods $\mu_{r}$ is estimated to be $10^{3} \mu_{\mathrm{B}}$ ( $\mu_{\mathrm{B}}$ is the Bohr magneton). The diamagnetic susceptibility $\Delta \chi$ at room temperature is $-1.7 \times 10^{-3}$ and the average particle volume $v_{0}=5.6 \times 10^{-23} \mathrm{~m}^{3}$. Using these numbers in Eq. (2), we obtain $J=2.28 \mathrm{~T}^{-1}$ and $K=0.72 \mathrm{~T}^{-2}$. These values need to be considered with some care because the magnetic properties are size dependent and the inherent size polydispersity of the system, therefore, leads to a considerable spread in $J$ and $K$.

To check whether these numbers are representative for the experimental goethite system, we may trace $S_{2}$ as a function 
of $B$ for a dilute (i.e., isotropic at zero field) system and locate its zero point. Beyond this point, the nematic order parameter is negative, which signifies a gradual change toward the planar-type nematic order found at high field strengths. Experimentally, the zero point is located at $0.35 \mathrm{~T}$, whereas theoretically we find $1.809 \mathrm{~T}$, irrespective of the density (at least in the paranematic density range, as will become clear later). The large discrepancy is attributed to the incertainty in $K$, which depends sensitively on the particle size. We can achieve much closer agreement by doubling this value such that $J=2.28 \mathrm{~T}^{-1}$ and $K=1.44 T^{-2}$. This gives a zero point at $0.552 \mathrm{~T}$, in reasonable agreement with the experimental value. In the actual calculations, we shall fix $J$ at $2.28 \mathrm{~T}^{-1}$ and vary $K$ to verify the sensitivity of the phase behavior with respect to a change of the (dia)magnetic properties.

\section{B. Electrostatic properties}

The double-layer potential around a charged colloid (with constant surface charge density) can be determined from the Poisson-Boltzmann (PB) equation which, in our case, must be solved for a cylindrical geometry. At large distances, the electrostatic potential $\psi$ around a cylinder with diameter $D$ takes the Debye-Hückel (DH) form [21]

$$
\beta \psi e=\Gamma K_{0}(\kappa D / 2),
$$

where $e$ is the elementary charge and $K_{0}$ a modified Bessel function. The proportionality constant $\Gamma$ depends upon the surface charge density $\sigma_{\mathrm{el}}$ of the particles. For highly charged particles like goethite, the linearized (DH) equation cannot be used to obtain $\Gamma$. Instead, the full (nonlinear) PB equation must be solved to determine its value. Approximate but accurate analytical solutions of the PB equation for a cylindrical geometry were obtained by Philip and Wooding [31], which allow for a straightforward calculation of $\Gamma$ by means of a simple iterative procedure. Once $\Gamma$ has been obtained, the effective rod diameter can easily be calculated from

$$
\frac{D_{\text {eff }}}{D}=1+(D \kappa)^{-1}\left\{\ln \left[\frac{\pi \Gamma^{2} \exp [-\kappa D]}{\kappa Q}\right]+\gamma_{E}-(1 / 2)\right\},
$$

where $\gamma_{E}$ is Euler's constant and $Q$ the Bjerrum length. Using $\sigma_{\mathrm{el}} \approx 0.2 \mathrm{C} / \mathrm{m}^{2}, D \approx 15 \mathrm{~nm}$, and ionic strength $I \approx 4$ $\times 10^{-2} \mathrm{M}$, we find $\kappa^{-1} \approx 1.5 \mathrm{~nm}$ and $\Gamma \approx 1.0 \times 10^{3}$ for the goethite rods. Equation (13) then gives us $D_{\text {eff }} / D \approx 1.65$. For the twisting parameter, we thus find $h \approx 0.063$. These results indicate that the effect of twist is expected to be rather insignificant. The diameter ratio however is quite high so that the effective aspect ratio $L / D_{\text {eff }}$ of the spherocylinder is much smaller than that of the bare particle. Throughout this study, we use $L / D=10$, which is a reasonable estimate of the average aspect ratio of the goethite rods [18], so that $L / D_{\text {eff }}$ $=6.3$ and $\phi_{\mathrm{eff}} / \phi_{\mathrm{HC}}=2.83$, with $\phi_{\mathrm{HC}}$ being the hard-core volume fraction.

The isotropic-nematic transition densities at zero field are $\phi_{\mathrm{HC}}^{(I)}=0.125$ and $\phi_{\mathrm{HC}}^{(N)}=0.132$. Comparing these with the experimental phase boundaries, $\phi_{\mathrm{HC}}^{(I)}=0.055$ and $\phi_{\mathrm{HC}}^{(N)}=0.085$

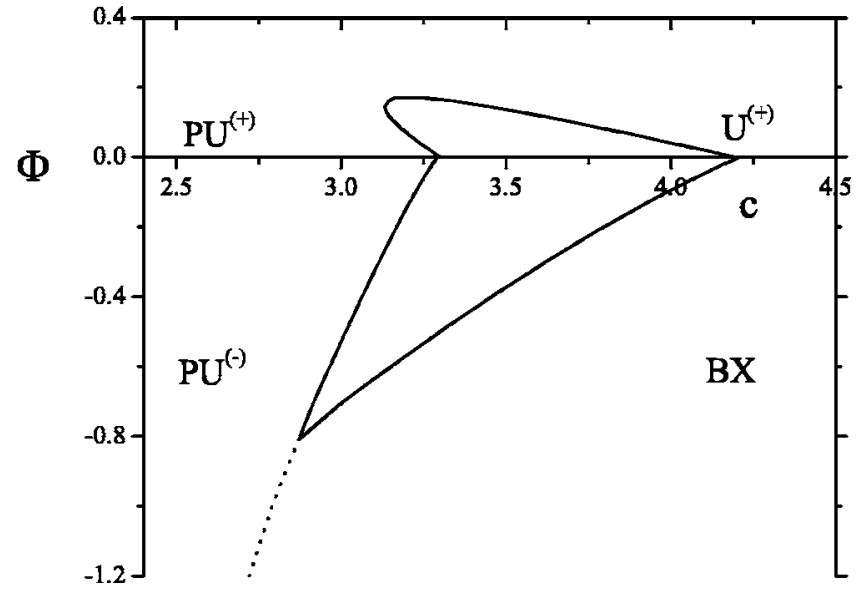

FIG. 1. Generalized para-nematic-nematic phase diagram for rods in external directional fields; $\Phi>0$ in the case of an orientational field, $\Phi<0$ for a disorientational field. Para-nematic-nematic binodals are given by solid lines, the dotted one represents a second-order phase transition from the para-uniaxial to the biaxial nematic state.

[18], shows a large overestimation which indicates that our simple model cannot provide quantitative results for goethite. The large density gap found in experiment is mainly due to the particles' considerable size polydispersity and seems to be a generic phenomenon in polydisperse systems [32].

\section{RODS IN DIRECTIONAL EXTERNAL FIELDS: GENERAL SCENARIOS}

Before discussing the complex phase behavior of the model goethite systems, we shall first present two simple general scenarios which occur if rodlike particles are subjected to an external directional electromagnetic field. The general phase diagram for this case has been presented in Fig. 1. This diagram is qualitatively similar to the one constructed by Khokhlov and Semenov in Ref. [12] and has been recalculated here for infinitely thin hard rods $(L / D$ $\rightarrow \infty, h=0$ ) based on the free energy Eq. (3). For convenience, the external energy Eq. (1) has been replaced by

$$
\beta U_{\text {ext }}(\cos \theta)=-\Phi \mathcal{P}_{2}(\cos \theta)
$$

in terms of a general field parameter $\Phi$ with $\theta$ the angle between the rod main axis and the field direction. If $\Phi>0$, the rods prefer to align along the field, and common "polar" nematic order occurs [indicated by "(+)"]. In this case, the field is referred to as having an "orientational" effect on the rods [12]. Note that due to the Boltzmann factor, $\exp \left[-\beta U_{m}\right]$, in Eq. (7), the isotropic state no longer exists at finite field strengths. Instead, dilute systems now show weakly aligned para-nematic order, indicated by "P." Both nematic phases are of uniaxial symmetry and the first-order para-nematic-nematic coexistence region terminates in a critical point above which the system changes from one state to the other in a continuous fashion. As to magnetic fields, the present scenario may be observed for, e.g., rods with a 
positive magnetic susceptibility, $\Delta \chi>0$, leading to an induced moment along the main rod axis. The magnetic field then gives rise to liquid crystalline order of the orientationalquadrupolar type [12]. Obviously, similar behavior is expected for rods with a permanent magnetic dipole moment along their main axis, like goethite (orientational dipolar field). In this case one refers to orientational-dipolar-type order.

In the opposite case $(\Phi<0)$ the field has a "disorientational" effect and the rods preferentially orient in a plane perpendicular to the field. Both para-nematic and nematic phases are now of the planar, or antinematic type, indicated by "(-)." Moreover, in the concentrated nematic phase, the rods apparently pack more favorably if they attain an additional direction of alignment within the plane. The nematic phase, thus, has a biaxial symmetry. For $\Phi<0$, the first-order para-nematic-nematic transition terminates in a tricritical point [14]. Beyond this point, the transition from one state to the other occurs by means of a second-order phase transition. The present type of ordering may occur if the rods have a negative diamagnetic susceptibility anisometry so that the induced magnetic moments are perpendicular to their main axes (disorientational quadrupolar order). This is the case for the induced moments of the goethite rods. Similar behavior has been found recently for systems of colloidal gibbsite platelets in a magnetic field, where magnetic moments are induced along the short axis of the particle [33].

It is clear from the above that the goethite systems are expected to display characteristics from both scenarios. This will become clear in the next section where we shall discuss some explicit phase diagrams for our model goethite systems.

\section{PHASE DIAGRAMS FOR GOETHITE}

\section{A. Quadrupolar scenario}

Figure 2(a) shows a phase diagram for "goethite" spherocylinders. This scenario is similar to the experimental situation, judging from the location of the dashed line which marks the gradual change from polar-type to planar-type nematic order in the dilute regime, discussed in Sec. III A. We refer to this diagram as the "quadrupolar scenario" since the high-field region of the diagram is largely determined by the induced magnetic moments. Hence, the appearance is similar to the disorientational quadrupolar scenario depicted in Fig. 1.

At low field strengths, the remanent moments dominate and the diagram is governed by the orientational effect of the field, i.e., the para-nematic and nematic states are both uniaxial and the rods are strongly aligned along the field direction. However, upon increasing the field strength, the degree of polar order will decrease since the induced moments (perpendicular to the main rod axis) become more pronounced. At some point, the uniaxial nematic state changes to a biaxial one and a first-order (para-)uniaxialbiaxial (PU-BX) nematic coexistence develops. The coexistence region eventually narrows down toward a tricritical point, beyond which the $P U^{(-)}-B X$ transition becomes second order [12]. At very high field strengths, the induced moments
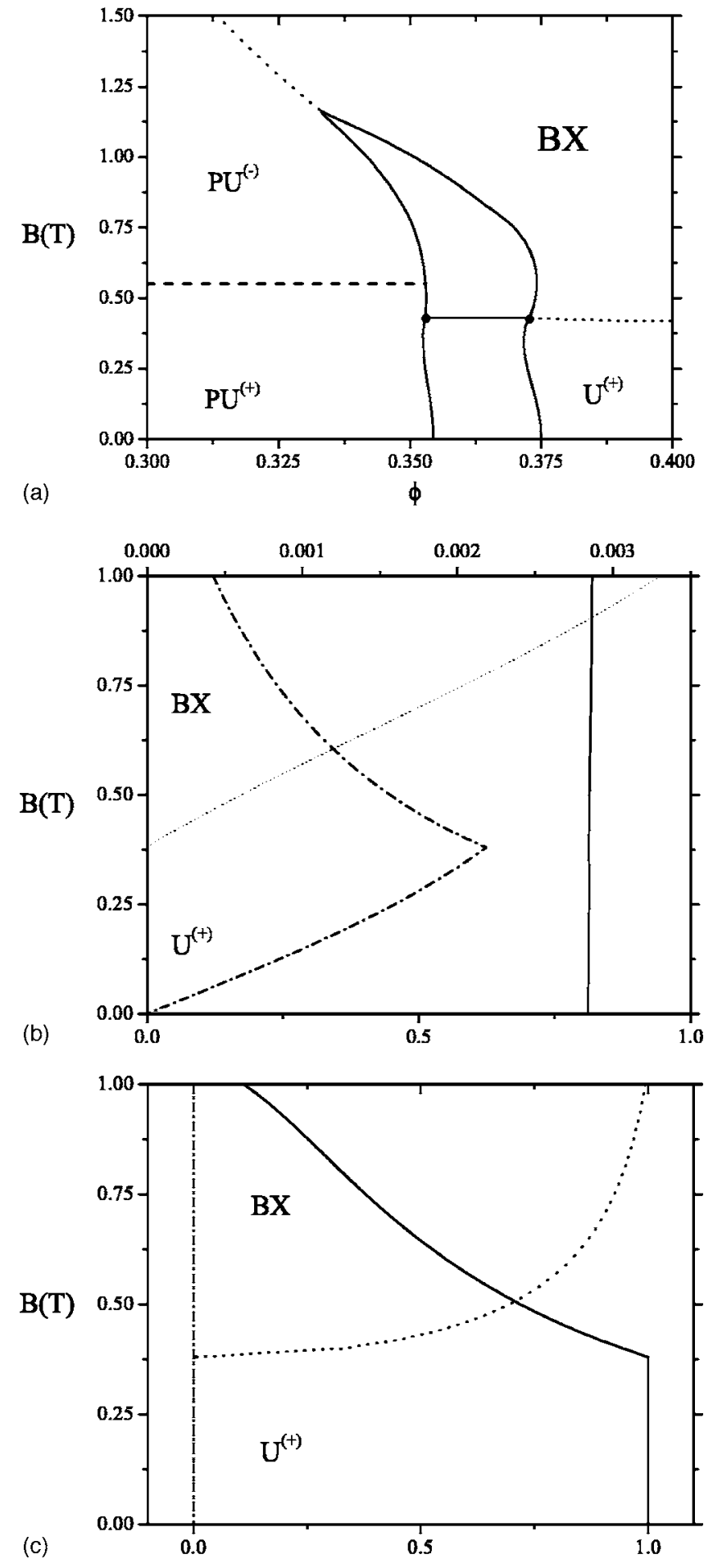

FIG. 2. (a) Phase diagram of goethite spherocylinders with $J$ $=2.28 \mathrm{~T}^{-1}, K=1.44 \mathrm{~T}^{-2}$, and $L / D_{\text {eff }}=6.3(L / D=10)$. Binodals are indicated by solid lines. The dashed line in the para-nematic region corresponds to $S_{2}=0$. The dotted curves denote second-order phase transitions from the uniaxial $(P) U$ to the biaxial $B X$ nematic state. The corresponding behavior of the nematic order parameters, $S_{1}$ (dash-dotted line), $S_{2}$ (solid line), and $\Delta$ (dotted line) is shown in (b) for $\phi=0.40$. The values for $\Delta$ are indicated on the upper horizontal axis. (c) Nematic director in terms of its projection on the $x$ axis (dotted line), $y$ axis (dash-dotted line), and $z$ axis (solid line) of the laboratory frame. The magnetic field is parallel to the $z$ axis. 
will completely outweigh the remanent ones and force the rods to orient almost perfectly in a plane perpendicular to the field. The planar $P U-B X$ bifurcation then becomes reminiscent of a quasi-two-dimensional isotropic-nematic transition [14].

Let us now focus on the field-induced transitions corresponding to the homogeneous (monophasic) systems, given by the horizontal curves in Fig. 2(a). In the dilute regime, the ODF changes continuously from polar-type (peaked around $\theta=0$ ) to planar-type (peaked around $\theta=\pi / 2$ ) and the transition can be roughly localized from the condition $S_{2}=0$ (dashed curve). Note that the corresponding curve does not represent a phase transition, it merely localizes a gradual change of signature of the ODF. The curve is virtually independent of the packing fraction since the ODF in the dilute regime is mainly determined by the Boltzmann factor in Eq. (7).

In the concentrated regime, there is a continuous transition from the uniaxial to the biaxial nematic state. As is clear from Fig. 2(c), the transition to the biaxial nematic state coincides with the field strength where the nematic director starts to turn away from the field direction. Obviously, if the nematic director is not parallel to the field, the azimuthal rod projections (perpendicular to the director) are no longer equally favorable and the uniaxial symmetry of the nematic state is lost. At larger field strengths, the degree of biaxiality increases as the nematic director gradually rotates perpendicular to the field. The reorientation of the nematic director has also been observed in experiment, albeit at a somewhat lower applied field, $B \sim 0.2 \mathrm{~T}[19]$. We remark that the biaxial nematic order parameter $\Delta$ remains very small in the experimentally accessible field range. Experimental studies of electric field effects in thermotropic nematic systems have revealed similar weak biaxiality occuring even at high field strengths $[34,35]$.

It is clear from Fig. 2(b) that the rods remain sufficiently ordered along their main directors throughout the entire field range. By gradually rotating its main nematic director perpendicular to the field, the system is able to sustain the level of polar nematic order without changing to planar nematic order such as in the dilute regime. This particular property allows us to perform a simple analysis of the free energy, valid for strongly aligned, near-parallel orientational configurations. In the Appendix, we show that the realignment behavior can then be described in terms of a simple spin concept, in which the rods behave as ideal spins pointing either parallel or antiparallel to the field.

\section{B. Dipolar scenario}

If we reduce the diamagnetic susceptibility anisometry (by lowering $K$ ) the diamagnetic effect becomes relatively unimportant at low fields. The influence of the remanent dipole moments is then expected to govern the phase behavior in this regime. We see from Fig. 3 that the topology is indeed very similar to the orientational scenario of Fig. 1. The paranematic-nematic coexistence region terminates in a critical point above which the system changes gradually from one state to the other without any discontinuity or jump in the associated nematic order parameters.

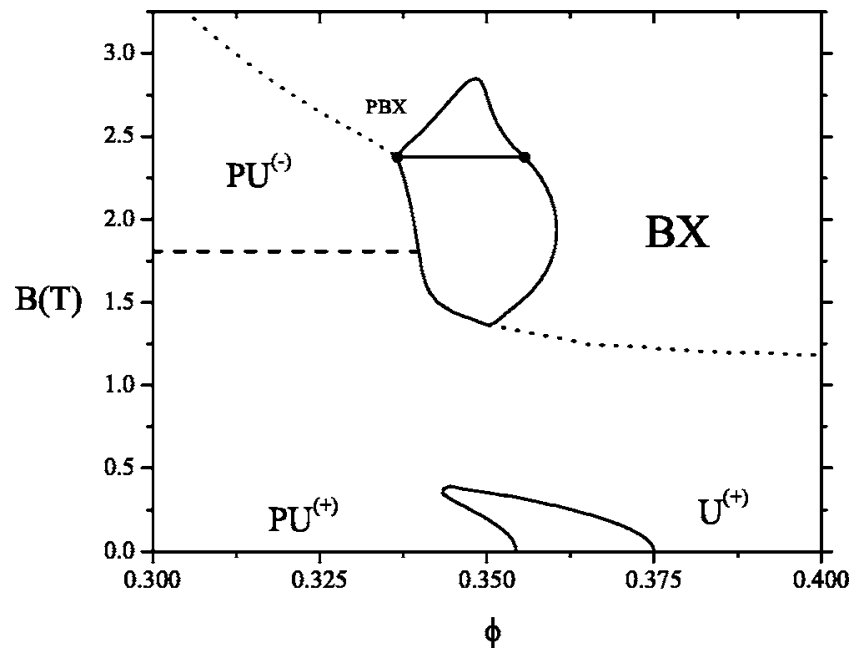

FIG. 3. Phase diagram for $J=2.28 \mathrm{~T}^{-1}$ and $K=0.72 \mathrm{~T}^{-2}$. All dotted curves denote second-order phase transitions from the uniaxial to the biaxial nematic state.

At high field strength, the induced moments become dominant and give rise to a reopening of the phase gap beyond some critical $B$ value. Note that the nematic phase is now of biaxial symmetry, like in Fig. 2(a). A remarkable difference with the previous scenario, however, is that the para-nematic phase becomes biaxial as well at $B>2.38 \mathrm{~T}$. In this regime, a coexistence between two biaxial phases [a para-biaxial nematic $(P B X)$ and a biaxial nematic $(B X)$ one] develops which eventually closes off at a critical or consolute point located at $B=2.848 \mathrm{~T}$. Beyond this point, the system gradually changes from one state to the other, similar to the para-nematic-nematic transition above the $P U^{(+)}-U^{(+)}$ critical point. In fact, the para-biaxial-biaxial demixing region can be considered as the high-field analog of the $P U^{(+)}-U^{(+)}$transition. Both involve a coexistence between phases of equal symmetry and the entropic mechanism underpinning the demixing is governed by a competition between orientational entropy (favoring the weakly ordered para-nematic state) and packing entropy (favoring the nematic state).

An obvious consequence of reducing $K$ is that the transitions pertaining to the homogeneous systems shift to much higher $B$ values, as we see in Fig. 3. In the concentrated regime, the transition from the uniaxial to the biaxial state is continuous and corresponds to the point where the rods start to collectively turn away from the field direction, similar to the situation indicated in Fig. 2(b) and 2(c).

\section{Intermediate scenario}

If we choose an intermediate value for $K$ we get an even richer phase diagram as can be seen from Fig. 4. Close inspection reveals that the diagram contains features of both previous scenarios. This is particularly notable at high field strengths, where we observe two para-nematic-nematic regions (involving a $P U-B X$ and a $P B X-B X$ coexistence) reminiscent of the upper regions of Fig. 2(a) and Fig. 3, respectively. Upon lowering $B$, the two regions meet at a triple line, 


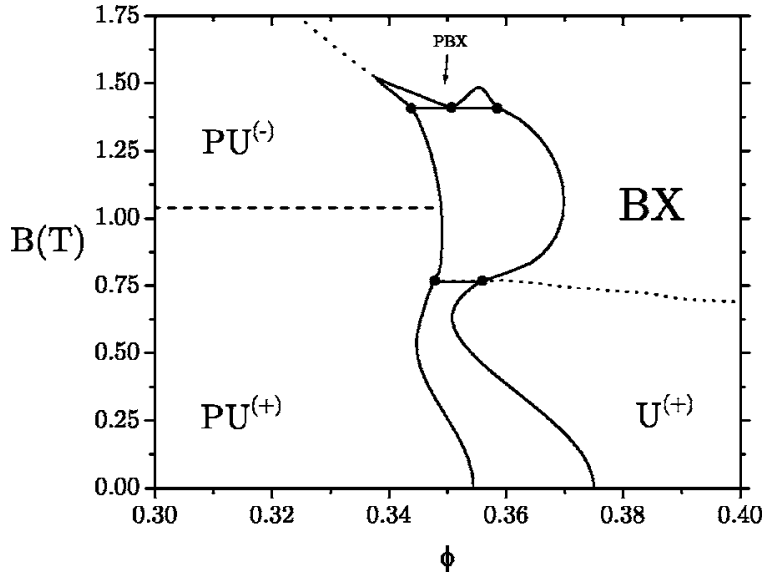

FIG. 4. Phase diagram for $J=2.28 \mathrm{~T}^{-1}$ and $K=1.08 \mathrm{~T}^{-2}$. All dotted curves denote second-order phase transitions from the uniaxial to the biaxial nematic state. A triple line is located at $B$ $=1.41 \mathrm{~T}$

indicating a triphasic coexistence between a uniaxial nematic phase and two biaxial nematic phases each with a different concentration. Marked reentrant and remixing effects are notable around $\phi=0.36$ where a sequence of phase coexistences may be expected upon increasing field strengths.

The present scenario can be nicely connected to the previous ones by focussing on the triple equilibrium. If we increase $K$, the concentration of the para-biaxial phase (middle dot) is expected to move closer to that of the coexisting biaxial phase (right dot) so that the biaxial-biaxial region is pushed out of the diagram. At some $K$ value, both concentrations meet at a critical end point where the $P B X-B X$ region has completely disappeared. From this point on the scenario will be similar to Fig. 2(a). If we decrease $K$, the opposite happens: the uniaxial-biaxial region is squeezed out at the benefit of the biaxial-biaxial region (Fig. 3). Simultaneously, the lower $P U^{(+)}-U^{(+)}$binodals detach from the upper $P U^{(-)}-B X$ ones. The latter now constitute a separate coexistence region, enclosed by a lower tricritical point and an upper consolute point.

\section{CONCLUSIONS}

Within the Onsager-Parsons theory, we have investigated the stability of the various nematic states which may appear in systems of goethite rods when subjected to an external magnetic field. In the present study, the goethite rods are represented by charged spherocylinders bearing a remanent magnetic moment (leading to preferred dipolar order) and a negative diamagnetic susceptibility anisometry (leading to preferred planar, or quadrupolar, order). These mixed dipolar/quadrupolar properties give rise to intricate liquid crystalline phase behavior. Depending on the relative contributions of the particles' remanent dipole moment and the negative magnetic susceptibility anisometry, several scenarios were constructed. The quadrupolar scenario, in which the effect of the remanent moments is relatively small, is characterized by a gradual reorientation of the nematic director at some critical field strength. The collective rotation of the rods away from the magnetic field direction has been observed in x-ray scattering studies of concentrated goethite samples. We have shown that the realignment phenomenon can be described qualitatively by a simple spin concept, which allows us to approximately locate the onset of the director realignment in terms of a second-order phase transition.

Similar reorientation phenomena occur upon lowering the susceptibility anisometry, but the phase diagrams become drastically different in these cases. In the dipolar scenario, where diamagnetic effects only become manifest at high field strengths, two separate para-nematic-nematic coexistence regions are found at low and high fields, the latter involving two biaxial nematic phases. At intermediate susceptibilities, a triphasic coexistence is found between a uniaxial paranematic phase and two biaxial nematic ones.

In the present calculations, we have not accounted for the bar-shaped geometry of the goethite particles. The inherent biaxial shape may have serious consequences for the phase diagrams presented here, in particular with respect to transitions to the biaxial nematic state. We anticipate that biaxial order will be significantly stabilized because the bar shape makes them prone to biaxial nematic order, even at zero field. Further complications, such as size polydispersity could also be addressed by considering, e.g., binary mixtures of two different-sized spherocylinders. Note that the size dependency of the magnetic properties should then also be taken into account. However, given the complex phase behavior of the monodisperse systems considered here, one may question whether it is worthwhile to pursue this direction.

From an experimental point of view, a promising way to reconciliate the present model system with the experimental one could be to reduce both the intrinsic bar shape and the size spread of the colloids. The first could be achieved by coating the particles with a layer of silica which would render them more cylinder-like. The coating procedure also opens up the possibility of introducing hard-particle interactions by applying a polymer grafting of the silica-coated particles and redispersing them into a suitable apolar solvent. However, we do not expect this modification to give significantly different phase behavior since the electrostatic twist effect is of marginal importance and all phase diagrams presented here qualitatively apply to "hard" goethite rods as well. The second goal, reduction of the polydispersity, can be reached using various purification and fractionation methods. In particular, reducing the particles' considerable length polydispersity would be desirable to enhance the stability of smectic order. Finally, a systematic variation of particle size is expected to influence the relative importance of permanent and induced magnetic moments, which would offer a means to address different theoretical scenarios. These experimental topics are currently under investigation at the Van 't Hoff laboratory and significant progress has already been made.

\section{ACKNOWLEDGMENT}

We want to thank Patrick Davidson for stimulating our interest in this problem. 


\section{APPENDIX: SPIN MODEL}

If the spherocylinders are perfectly aligned, the uniaxial state $(\mathrm{U})$ is represented by a collection of rods all (anti)parallel to the field direction. In the "tilted" biaxial phase (BX), the rod dipoles make an angle $\beta$ or $\pi-\beta(0<\beta \leqslant \pi / 2)$ with the field direction. At fixed concentration, the excludedvolume entropy $\rho$ (for $\gamma \equiv 0$ ) is identical in both states and the interactions, therefore, do not contribute to the free energy difference. Conceptually, the system can be considered as a system of ideal spins. In the $U$ state, the magnetic energy of a single spin is then equal to $-J B+K B^{2}$ (up, parallel to the field) or $J B+K B^{2}$ (down, antiparallel), according to Eq. (1). Similarly, in the BX state, we substitute $\theta=\beta$ (up) and $\theta=\pi-\beta$ (down) in Eq. (1) for all particles. The free energy difference $\Delta \mathcal{F}(\beta)=\mathcal{F}_{B X}-\mathcal{F}_{U}$ is now easily calculated from the spin partition function and reads

$$
\Delta \mathcal{F}(\beta)=\ln \left[\frac{\cosh J B}{\cosh (J B \cos \beta)}\right]+\frac{3}{2} K B^{2}\left(\cos ^{2} \beta-1\right) .
$$

Taking the derivative of the free energy with respect to $\beta$ reveals that the uniaxial nematic state (i.e., $\beta=0$ ) is a trivial solution of the stationarity condition $\partial \Delta \mathcal{F} / \partial \beta=0$ at all field strengths. At high fields, this equation will have nonzero solutions $\beta \neq 0$ indicating a possible transition to the "tilted" biaxial state. The branching point is found by performing a simple Landau expansion of Eq. (A1) in terms of the "order parameter" $\beta$. Expanding up to the fourth order in $\beta$ gives

$$
\Delta \mathcal{F}(\beta)=C_{1} \beta^{2}+C_{2} \beta^{4}+O\left(\beta^{6}\right)
$$

with

$$
\begin{gathered}
C_{1}=\frac{1}{2}\left(J B \tanh J B-3 K B^{2}\right), \\
C_{2}=\frac{1}{24}\left\{12 K B^{2}-3(J B)^{2} \cosh ^{-2} J B-J B \tanh J B\right\} .
\end{gathered}
$$

Note that Eq. (A2) does not have a cubic contribution $\left(\propto \beta^{3}\right)$ since $\beta$ and $-\beta$ are thermodynamically equivalent. Furthermore, $C_{2}$ is generally positive indicating that we are dealing with a (continuous) phase transition of the second kind [36]. The tilting transition, in terms of the critical field strength $B_{c}$, can be determined from $C_{1}=0$. For the system in Fig. 2 this gives $B_{c}=0.350 \mathrm{~T}$; for the one corresponding to Fig. 3, we get $B_{c}=1.037 \mathrm{~T}$; and for Fig. $4, B_{c}=0.627 \mathrm{~T}$. The agreement with numerical results is reasonable, in particular at high packing fractions. In all three cases, the spin model seems to underestimate the uniaxial-biaxial nematic transition by about $15 \%$ (at $\phi=0.4$ ) compared to the numerical results. Larger discrepancies occur at lower concentrations mainly due to the neglect of subtle changes in the orientational and packing entropy at the tilting transition.

An analytical estimate of the critical field strength can be obtained if $J B_{c}$ is much larger than unity. In that case, the hyperbolic functions in Eq. (A3) are either close to zero or unity. From $C_{1}=0$, we then obtain the simple result $B_{c}$ $\sim J / 3 K$ (with $J \gg K$ ). Elaborating Eq. (A2) a little further reveals that

$$
|\beta| \sim \frac{1}{9 K^{2}}\left(\frac{B_{c}-B}{B}\right)^{1 / 2}
$$

close to the critical point, showing that the tilt angle obeys common mean-field scaling behavior [36].
[1] R. Evans, Adv. Phys. 28, 143 (1979).

[2] R. van Roij, M. Dijkstra, and R. Evans, Europhys. Lett. 49, 350 (2000).

[3] M. P. Allen, J. Chem. Phys. 112, 5447 (2000).

[4] K. Shundyak and R. van Roij, J. Phys.: Condens. Matter 13, 4789 (2001).

[5] L. Harnau and S. Dietrich, Phys. Rev. E 65, 021505 (2002).

[6] V. A. Baulin and A. R. Khokhlov, Phys. Rev. E 60, 2973 (1999).

[7] V. A. Baulin, J. Chem. Phys. 119, 2874 (2003).

[8] H. H. Wensink and H. N. W. Lekkerkerker, Europhys. Lett. 66, 125 (2004).

[9] M. Schmidt, M. Dijkstra, and J. P. Hansen, Phys. Rev. Lett. 93, 088303 (2004).

[10] S. V. Savenko and M. Dijkstra, Phys. Rev. E 70, 051401 (2004).

[11] L. Onsager, Ann. N.Y. Acad. Sci. 51, 627 (1949).

[12] A. R. Khokhlov and A. N. Semenov, Macromolecules 15, 1272 (1982).

[13] S. Varga and I. Szalai, Phys. Chem. Chem. Phys. 2, 1955 (2000).
[14] S. Varga, G. Kronome, and I. Szalai, Mol. Phys. 14, 911 (2000).

[15] S. Varga and I. Szalai, Mol. Phys. 93, 377 (1998).

[16] H. Graf and H. Löwen, J. Phys.: Condens. Matter 11, 1435 (1999).

[17] B. J. Lemaire, P. Davidson, J. Ferré, J.-P. Jamet, D. Petermann, P. Panine, I. Dozov, D. Stoenescu, and J. P. Jolivet, Faraday Discuss. 128, 271 (2005).

[18] B. J. Lemaire, P. Davidson, J. Ferré, J. P. Jamet, D. Petermann, P. Panine, I. Dozov, and J. P. Jolivet, Eur. Phys. J. E 13, 291 (2004).

[19] B. J. Lemaire, P. Davidson, J. Ferré, J. P. Jamet, P. Panine, I. Dozov, and J. P. Jolivet, Phys. Rev. Lett. 88, 125507 (2002).

[20] B. J. Lemaire, PhD thesis, Université Paris-Sud, (2002) (unpublished).

[21] A. Stroobants, H. N. W. Lekkerkerker, and T. Odijk, Macromolecules 19, 2232 (1986).

[22] T. Odijk, J. Chem. Phys. 93, 5172 (1990).

[23] S. L. Brenner and V. A. Parsegian, Biophys. J. 14, 327 (1974).

[24] J. D. Parsons, Phys. Rev. A 19, 1225 (1979).

[25] S. D. Lee, J. Chem. Phys. 87, 4972 (1987). 
[26] S. D. Lee, J. Chem. Phys. 89, 7036 (1989).

[27] N. F. Carnahan and K. E. Starling, J. Chem. Phys. 51, 635 (1969).

[28] J. Herzfeld, A. E. Berger, and J. W. Wingate, Macromolecules 17, 1718 (1984).

[29] H. H. Wensink, G. J. Vroege, and H. N. W. Lekkerkerker, Phys. Rev. E 66, 041704 (2002).

[30] M. P. Allen, G. T. Evans, D. Frenkel, and B. M. Mulder, Adv. Chem. Phys. 86, 1 (1993).

[31] J. R. Philip and R. A. Wooding, J. Chem. Phys. 52, 953 (1970).
[32] P. Sollich, J. Phys.: Condens. Matter 14, 79 (2002).

[33] D. van der Beek, A. V. Petukov, H. H. Wensink, G. J. Vroege, P. Davidson, W. Bras, and H. N. W. Lekkerkerker (unpublished).

[34] D. A. Dunmur, K. Szumilin, and T. F. Waterworth, Mol. Cryst. Liq. Cryst. 149, 385 (1987).

[35] J. A. Olivares, S. Stojadinovic, T. Dingemans, S. Sprunt, and A. Jákli, Phys. Rev. E 68, 041704 (2003).

[36] L. D. Landau and E. M. Lifschitz, Statistical Physics, Part I. (Butterworth, Oxford, UK, 1980). 